\title{
PULSATIONS OF OB-STARS: NEW OBSERVATIONS
}

\author{
DIETRICH BAADE \\ European Southern Observatory \\ Karl-Schwarzschild-Str. 2 \\ D-85748 Garching, Germany \\ Internet:dbaade@eso.org
}

\begin{abstract}
Improved observing and data analysis strategies have initiated a considerable expansion of the empirical knowledge about the pulsations of OB stars. Possible correlations between physical parameters and associated pulsation characteristics are becoming more clearly perceivable. This starts to include the asteroseismologically fundamental areas of $g$-modes and rapid rotation. The $\beta$ Cephei instability strip continues to be the only locus where radial pulsations occur (but apparently not in all stars located in that strip). Except for spectral types B8/B9 near the main sequence, where pulsations are hardly detected even at low amplitudes, any major group of stars in the Galaxy that are obviously not candidate pulsators still remains to be identified. However, the incidence and amplitudes of OB star pulsations decrease steeply with metallicity. The behaviour of high-luminosity stars is less often dominated by very few modes. In broad-lined stars the moving-bump phenomenon is more common than low-order line-profile variability. But its relation to nonradial pulsation is not clear. The beating of low- $\ell$ nonradial pulsation modes that have identical angular mode indices may be the clockwork of the outbursts of at least some Be stars. The physics of this episodic mass loss process remains to be identified.
\end{abstract}

\section{Introduction}

Ever since their discovery, pulsating OB stars have suffered from the lack of theoretical permissibility. The eventual confirmation through improved opacity calculations of the $\kappa$ mechanism as a viable driving mechanism (cf. Dziembowski, these proceedings) has given the field a considerable impetus. It has led to the very successful exploitation during the past couple of years of (i) multi-target databases resulting from spacecraft observations (Hipparcos) or from the monitoring of clusters with CCD's, (ii) very long 
time series of high-resolution spectra, (iii) improved techniques for their analysis, and (iv) a larger wavelength coverage of the observations.

The results open new perspectives for the study of the actual physical processes. Some groups of stars, such as supergiants and Be stars, which originally were defined on the basis of persistent spectroscopic differences and inferred physical properties, can also by means of their pulsation properties be distinguished. This is intriguing because it gives rise to the question of a possible causal relationship between pulsational and other properties and its directionality.

After short reviews of the properties of all known groups of pulsating OB stars (Sects. 2-5), an attempt is made to summarize the results in terms of physical parameters and to identify areas where theoretical support could be useful (Sect. 6).

\section{2. $\beta$ Cephei Stars}

For $\beta$ Cephei stars, a convenient defining criterion remains the shortness of their periods which range from 2 to 6 hours. Only in $\beta$ Cephei stars, but not all of them, have radial modes so far been detected. Since the nonradial modes are low-degree $p$-modes, the $\beta$ Cephei stars not only form a compact group in spectral type (B0.5B2), but are isolated from other pulsating OB stars also in a parameter space defined by period and mode indices. In careful analyses of significant numbers of high-quality spectra of several stars, Aerts and collaborators (e.g., 1995) have re-classified the types of known modes and also detected additional ones. Recent mainly photometrically oriented reviews are by Sterken \& Jerzykiewicz (1993) and Sterken (1993).

The knowledge about $\beta$ Cephei stars has also benefitted much from the use of array detectors for the photometry of young stellar clusters in Galaxy (Balona et al. 1997), LMC (e.g., Balona 1992), and SMC (Kjeldsen \& Baade, in preparation). The Galactic-cluster work showed that with the new opacities, the theoretical position of the $\beta$ Cephei instability strip matches the observed one quite well. Balona et al. (1997) also develop an interesting additional application of the study of $\beta$ Cephei pulsators: Because during their evolution B stars of different masses enter the instability strip at different ages, but also have different eigenfrequencies, the pulsation periods of $\beta$ Cephei stars turn out to be surprisingly good cluster age indicators.

At one-half solar metallicity, Balona $(1992,1993)$ found no candidate $\beta$ Cephei stars in LMC clusters. This is consistent with the need of the $\kappa$-mechanism for sufficient opacity to drive oscillations (cf. Dziembowski, these proceedings). However, in younger low-metallicity clusters with more massive stars in the instability strip, $\beta$ Cephei stars are not excluded by theory. The tentative identification of a 4.3- $\sigma$ candidate variable star (and several other $\geq 3 \sigma$ stars) in the probably even more metal-poor SMC cluster NGC 330 by Kjeldsen \& Baade (in preparation) is, therefore, not necessarily in conflict with theory. However, the period of somewhat less than 2 hours would be very short. Furthermore, the peak-to-peak $b$-band amplitude is only $0.003 \mathrm{mag}$, i.e. well below Balona's detection threshold of $\sim 0.01 \mathrm{mag}$.

In the name-giving $\beta$ Cephei itself a manifold of pecularities has been found which is so amazing (for references see Hadrava \& Harmanec 1996 and Telting et al. 1997) that either it must serve as a warning how ill-understood $\beta$ Cephei stars are in general or $\beta$ Cephei actually is the proto-type of a quite different class of variables. After the discovery many years ago of a presumably cyclic modulation of the wind and of a magnetic field of several $100 \mathrm{G}$, new polarimetry and UV-spectroscopy have been used to suggest an oblique magnetic rotator model with period 12 days. 
However, the evidence of genuine periodicity is weak. In particular, since only very few cycles are covered, the apparent double-wave variability may only be due to a variable amplitude. In this case the true wind and magnetic-field period, if any, would be 6 days. Furthermore, $\beta$ Cep is the primary in an eccentric binary with 90 -year period, and there are extended phases of $\mathrm{H} \alpha$ line emission. The wings of the $\mathrm{H} \alpha$ emission line vary in radial velocity with the period of the single radial mode.

Telting et al. (1997) find in addition to that radial mode four more frequencies. Three of these four and the radial mode are separated by $1 / 6 \mathrm{c} / \mathrm{d}$ corresponding to one half of the claimed double-wave period of 12 days. This is perhaps the strongest evidence to date of such a period in $\beta$ Cephei, and there is a weak, but not (yet?) significant hint at a fifth frequency fitting that pattern. Shibahashi \& Aerts (these proceedings) present a magnetic pulsator model in which the magnetic field distorts the radial eigenmode such as to add a quadrupole component. The latter would reveal itself by an evenly spaced frequency quintuplet. The model requires that the frequency spacing is identical to the rotation frequency, i.e. the double wave behaviour inferred from polarization and stellar wind data would be wrong.

The study of the line-profile variability associated with the sixth mode, which probably is nonradial (Telting et al. 1997), could cast light on a putative oblique nonradial pulsator model. If the pulsation axis is not aligned with the rotation axis, the line-profile variability depends on the rotational phase. Constraints on the angle between rotation and magnetic axis and on $i$ can be derived from such a dependency.

Using EUVE and exploiting the particularly EUV-transparent line of sight to $\beta$ Canis Majoris, Cassinelli et al. (1996) could for the first time determine the temperature variations in the Lyman continuum. They are about $110 \mathrm{~K}$ for the primary mode and about $25 \mathrm{~K}$ for the other two. Comparison of the 3 velocity and temperature amplitudes and monitoring of the beating effects should enable a detailed analysis of the atmospheric response to (nonradial) $\beta$ Cephei pulsation to be made.

The disentangling of two almost resonating modes in $16 \mathrm{Lac}$ with a beat period of $\leq 2$ years by Jerzykiewicz \& Pigulski (1996) is perhaps the most impressive example to date as to how important long and homogeneously analysed time series are.

\section{53 Per, $\epsilon$ Per and Slowly Pulsating B Stars}

Various names are in use for the pulsating $B$ stars that are neither $\beta$ Cephei stars nor supergiants nor Be stars. But no systematic survey of the pulsational properties of this potentially very large group has been made, and the application of the nomenclature appears relatively arbitrary and not well tuned to the true pulsational heterogeneity of the stars in question which indeed seems to be emerging. The most comprehensive report on Slowly Pulsating B Stars is by Waelkens (1991); the 53 Per stars and $\epsilon$ Per were described by Smith (1981) and Gies (1991), respectively.

In the Hipparcos photometry, a large number of new Slowly Pulsating B Stars was detected (Waelkens et al. 1997; see also Aerts et al., these proceedings). All of them appear to fall on the red side of the $\beta$ Cephei instability strip and have a $v \sin i$ of less than $150 \mathrm{~km} / \mathrm{s}$. Since there are numerous B stars with larger projected rotational velocity (in the Bright Star Catalogue their proportion is about $25 \%$ of which only one-third are Be stars), this limit appears to be highly significant. This is all the more so as for the usually invoked sectoral NRP modes the amplitude is the largest on the equator, thus maximizing the detection probability at large $i$. Since photometry can only detect NRP modes with $\ell \approx m \leq 4$, this result is consistent with unpublished 
observations by Penrod (Smith \& Penrod 1984) and Baade (1987) of broad-lined $(v \sin i \geq 200 \mathrm{~km} / \mathrm{s}$ ) non-emission line B stars (Bn stars). In both studies large-scale line-profile variability was not detected whereas small-scale moving bumps are nearly ubiquitous.

The moving-bump phenomenon is also commonly found in OB stars within and blueward of the $\beta$ Cephei stability strip, one of the best studied cases being $\epsilon$ Per (B0.5 IV-III, Gies 1991; see also Howarth \& Reid 1993 for the O9.5 V star HD 93521). Its detection over a very wide range in effective temperature would furthermore render significant the restriction of the Hipparcos discoveries to effective temperatures lower than those of the $\beta$ Cephei stars. It would imply that in non-Be stars low- $\ell g$-modes mainly occur redward of the $\beta$ Cephei strip. On the other hand, low-degree $p$-modes seem to be present also in the hotter B stars. This follows from Smith's (1981) work on the 53 Per stars as candidates of which he preferentially selected narrow-lined early B-type stars. In fact, the lowest $|m|$ value found in $\epsilon$ Per, namely 3, (Gies 1991) makes this star a border-line case.

Obviously, $T_{\text {eff }}$ and $v \sin$ i appear to be governing some basic pulsation properties:

$-T_{\text {eff }} \leq T_{\text {eff, } \beta \text { Cep }}$ and $v \sin i<150 \mathrm{~km} / \mathrm{s}$ : high-order $g$-modes with $\ell \approx 2$. These are the Slowly Pulsating B Stars. The 53 Per stars are largely identical to them but the nature of the hotter candidate 53 Per stars deserves to be checked.

- $T_{\text {eff }} \leq T_{\text {eff }, \beta \text { Cep }}$ and $v \sin i \geq 200 \mathrm{~km} / \mathrm{s}$ : moving bumps without demonstrated general genuine periodicity; however, if due to NRP, at least some of their short periods would probably be indicative of $p$-modes. There is no obvious proto-type after which these stars could be named, and it is possible that they only form the hotter part of the following category.

$-T_{\text {eff }} \geq T_{\text {eff }, \beta \text { Cep }}$ : moving bumps and/or $p$ modes with $\ell \geq 4$. $\epsilon$ Per could plausibly be called the proto-type of this category.

This enumeration is complemented by the fact that among the stars with $v \sin i$ above $200 \mathrm{~km} / \mathrm{s}$ only the Be stars, which are found from late $\mathrm{O}$ to late $\mathrm{B}$ spectral types, seem to show low- $\ell$ line profile variability (which is probably due to high- $n g$-modes, see Sect. 5). It should be noted that all numerical thresholds given are crude estimates and only serve to illustrate the order of magnitude.

\section{Evolved, Luminous Stars}

Hipparcos contributed to the record of photometric variability also of OB supergiants (van Leeuwen et al. 1997, Waelkens et al. 1998). However, the diagnostic power of single-channel photometry for the understanding of the variability of these objects is limited and, perhaps, already exhausted. In particular, the variations in the wind and the photosphere are difficult to disentangle. Kaufer et al. $(1996,1997)$ have for several years closely monitored a few BA supergiants at high spectral resolution and with wide wavelength coverage. The time scales of both variabilities can exceed the one of the radial fundamental mode considerably. The wind-related variability probably largely exhibits rotationally induced aspect changes of a non-axisymmetric structure.

At the photospheric level, equivalent widths and the spectral energy distribution are constant (Kaufer et al. 1997). Moreover, the radial velocity variations do not depend on line strength, i.e. depth of formation. In some stars, even the movingbump phenomenon is seen and maintains phase coherence for at least a few cycles; often the acceleration of the bumps is too high to be due to rotation only. But the variable superposition of different variabilities makes it difficult to draw a more certain 
conclusion. This variability extends to some of the hottest not-subluminous stars known (e.g., $\zeta$ Pup: $T_{\text {eff }}=42,500 \mathrm{~K}$, Reid \& Howarth 1996).

All available observations are compatible with the kinematical effects of nonradial pulsation. However, contrary to low-luminosity stars, the inclusion of more data usually does not lead to a refinement of the temporal power spectrum. For as long as a numbner of years, the power spectra of many stars retain a certain overall signature which crudely distinguishes them from other stars. But the variations of individual features can already after few cycles be so pronounced that a single period is no longer sufficient to explain this behaviour.

\section{Be Stars}

Virtually all Be stars earlier than $\sim \mathrm{B} 8$ show low-order line-profile and/or photometric variability; attempts to associate these two variabilities with one another have at least in some cases been painful. Especially the spectroscopic variability seems to be the second criterion that distinguishes Be stars from other rapidly rotating B stars (cf. Sect. 3). The only well-known exception seems to be $\zeta$ Oph (Kambe et al. 1997) in which only the higher-order line-profile variability is seen that is common to nearly all broad-lined OB stars.

The periods are 0.5-2 days, remain stable over many years, and are statistically indistinguishable from the expected rotation periods. But a single, truly multiperiodic star would terminate the dispute (Baade \& Balona 1994) whether the variability of Be stars is due to some corotating stellar or circumstellar features or due to nonradial pulsation. However, so far only tentative, albeit intriguing, identifications of no more than two persistent periods have been possible in very few stars (e.g., $\zeta$ Oph: Kambe et al. 1997). Similarly, hopes that nonradial low- $\ell$ pulsation could be responsible for the seemingly irregular outbursts of many Be stars did not up to now materialize.

Recently, Rivinius et al. (1998) discovered in $\mu$ Cen six discrete frequencies in two groups: $f_{1} / f_{2} / f_{3} / f_{4}=1.9884 / 1.9704 / 2.0222 / 1.9366 \mathrm{c} / \mathrm{d}$ and $f_{5} / f_{6}=3.5536 / 3.5825$ $\mathrm{c} / \mathrm{d}$. Their phase proved coherent in four annual seasons spanning 5 years. The most fascinating result is that the beat periods of $f_{1}$ with $f_{2}$ and $f_{3}$ are also the mean intervals between line emission outbursts, i.e. discrete mass loss events. Since these three modes have the largest amplitudes in their group, apparently the vectoral co-addition of the two velocity fields must exceed a certain threshold for an outburst to occur. This explains that beating of, e.g., $f_{1}$ and $f_{5}$ is not important since for the $f_{1}-f_{4}$ group $\ell \approx 2$ whereas $\ell_{5} \approx \ell_{6} \approx 3$.

Outbursts observed within a decade of the data used by Rivinius et al. are with the same periods properly accounted for by this simple clock. Since this circumstellar activity can be described so well by using the photospheric periods only, the trustworthiness of the latter is underligned because the measurements of the photospheric radial velocities are independent of the circumstellar emission lines. It also suggests that there are no hidden $\ell \approx 2$ modes with amplitudes larger than the one of $f_{3}$.

In a Be star with a typical rotation frequency of $1 \mathrm{c} / \mathrm{d}$, the spacing of the $f_{1}-f_{4}$ group is too small by an order of magnitude to be due to $m$-mode splitting (besides, all four angular eigenfunctions look the same). Because the implied frequencies in the corotating frame are quite low, it is likely that these frequencies belong to $g$-modes with similar but different radial orders, $n$, and identical angular indices $\ell$ and $m$. Since the radial orders may be of order 100 and $\left|\Delta f_{2,4}\right| \approx\left|\Delta f_{1,3}\right| \approx 2 \times\left|\Delta f_{2,1}\right|, n_{1}$ and $n_{2}$ differ by one-half of the differences between $n_{1}$ and $n_{3}$ and between $n_{2}$ and $n_{4}$. One may, 
therefore, wonder whether between $f_{1}$ and $f_{3}$ as well as between $f_{2}$ and $f_{4}$ one mode each (more if $n_{1}$ and $n_{2}$ differ by more than unity) is either not excited or not detected. If the rotation period can be properly constrained, these frequency differences and the corotating frequencies should enable the first crude asteroseismological experiments with a rapidly rotating star or firmly rule out the NRP model for Be stars.

The difference between the $f_{1}-f_{4}$ and the $f_{5}-f_{6}$ frequencies, $\sim 1.56 \mathrm{c} / \mathrm{d}$, could plausibly be the rotation frequency. For a difference of unity in $m$ between the two groups, which inspection of the line profile variations does not rule out, rotational $m$-mode splitting is, therefore, a possibility. For the same reason, but if also $m=+\ell$ is adopted together with the above $\ell$ values, i.e. retrograde modes are assumed, all 6 eigenfrequencies would in the corotating frame be nearly identical. This would raise the question whether rotation plays a role in the mode selection.

The large number of variable Be stars in the LMC cluster NGC 330, which seem to have the same period distribution as Galactic Be stars (Balona 1992), would suggest that the driving of $g$-modes in these rapidly rotating stars is less sensitive to reduced metallicity than is the driving of radial and $p$-modes in $\beta$ Cephei stars.

\section{Physical Parameters}

The description in the previous sections of the pulsations of $\mathrm{OB}$ stars by types of objects, can tentatively be summarized in terms of physical parameters as follows:

Temperature: Outside the domain of the evolved, very luminous stars, incidence and/or amplitude of pulsations drop steeply at spectral types B8/B9. The pulsating OB stars are, therefore, clearly separated from the $\delta$ Scuti (Breger, these proceedings) and $\gamma$ Doradus (Krisciunas, these proceedings) variables. By contrast, a blue limit to this instability domain has not yet been established. It is not even clear whether there is any major population of Galactic OB stars in which also high-resolution spectroscopy would not find the signature of nonradial pulsations. It would be worthwhile investigating whether the chemically peculiar stars form such an exception. There is evidence that blueward of the $\beta$ Cephei instability strip low- $\ell$ NRP modes are less common than on the red side. The $\beta$ Cephei strip continues to be the only locus of radial and low-degree $p$-modes.

Metallicity: The incidence of $\beta$ Cephei pulsation apparently decreases strongly with metallicity. In LMC clusters, $\beta$ Cephei stars seem to be very much rarer than in the Galaxy. On the other hand, even in the SMC traces of radial pulsations may possibly occur. In marked contrast to this theoretically expected dependency, periodically variable $\mathrm{Be}$ stars are in some LMC clusters at least as common as in Galactic clusters and seem to have the same period distribution.

Age: Since more massive B stars reach the $\beta$ Cephei instability strip earlier, more massive $\beta$ Cephei stars are younger. The increase in the pulsation frequency of the radial fundamental mode with mass therefore leads to the more readily observable correlation between cluster age and pulsation period.

Rotation: Among rapid rotators $(v \sin i \geq 200 \mathrm{~km} / \mathrm{s}$ ) low- $\ell$ NRP modes seem to be common in Be stars only; their rotation and inertial-frame pulsation periods are very similar. Virtually all broad-lined B stars display higher-order line-profile variability. The latter looks similar to high- $\ell$ NRP modes but (the few) attempts to verify this identification more firmly have failed so far. With current observing techniques it is not possible to find out whether high-order line-profile variabil- 
ity occurs in narrow-line stars, too. It is an exciting possibility that the difficult subject of the theroretical treatment of rapid rotation may soon benefit from the incipient detection of multi-mode NRP in rapidly rotating stars. Because young clusters in LMC and SMC abound in periodically variable Be stars whereas $\beta$ Cephei stars are rare, it may be possible that rapid rotation helps (but is not sufficient) to destabilize high-order low-degree $g$-modes.

Luminosity: The variability of luminous OB stars is in most cases characterized by an apparently much more pronounced irregularity. However, evidence is mounting that the variability is due to multi-mode nonradial pulsation in high- $\boldsymbol{n} g$-modes with highly variable amplitudes. This would indicate that the ill-understood but very effective mode-selection mechanism, which apparently is acting in other candidate $g$-mode pulsators (Be and SPB stars), plays a much lesser role in supergiants.

Mass loss: In high-amplitude radial pulsators, IUE has not found significant alterations of the mass loss rate by the associated outward moving shock fronts. In $\mu$ Cen, the clock of its Be-star typical discrete mass loss events may finally have been identified: If the positive superposition of the velocity fields of two or more quadrupole modes exceeds a certain amplitude threshold, an outbursts occurs. In the case of more than two such modes also the long-term activity cycles of some Be stars may become reproducible. The physics of the outburst process, however, still remains unexplained.

Magnetic fields: The example of $\beta$ Cephei proves that a significant magnetic dipole field and bona fide $\beta$ Cephei pulsation are not incompatible with each other. If diffusion has created chemically distinct patches in the photosphere, the above conjecture of the CP stars being the only major exception to pulsational instability in OB stars would be challenged. The question of an oblique magnetic pulsator à la roAp stars should be answerable on the basis of the dependence or not of the line-profile variability on rotational phase. Perhaps more interestingly, $\beta$ Cephei is the only OB star known to date in which the effect of a strong magnetic field on the radial eigenmode can be studied.

It is important to remember that to most of the above 'rules' exceptions are known. But as guidelines, maybe even incentives, for further research they should be useful. Areas which observers should be curious to see commented on by theorists include:

- The occurrence of higher- $\ell$ modes towards higher temperatures than is the case for low-degree modes which are quite well reproduced by theory (cf. Dziembowski, these proceedings).

- The instability of the temporal power spectra of supergiants.

- The possibly inverse question of the very effective mode selection in lower-luminosity stars.

- The roughly 50:50 dichotomy (evolutionary bifurcation or consecutive stages?) among rapid rotators between $\mathrm{Bn}$ stars that do not show low-degree $g$-mode pulsations and $\mathrm{Be}$ stars that (i) do so and (ii), perhaps, only as a result of multi-mode beating eject matter, thereby possibly acquiring the circumstellar disk which actually makes them become Be stars.

- The unabated persistence of this phenomenon even at LMC metallicities.

- The confrontation of the observed frequencies of $\mu$ Cen with model calculations.

- The physics of the apparently pulsationally clocked outburst mechanism of $\mu$ Cen. 
Acknowledgement: I thank Petr Harmanec, Don Kurtz, Thomas Rivinius, Myron Smith, and Stanislav Stefl for valuable suggestions for improvements.

\section{References}

Aerts, C., Mathias, P., Van Hools, T., De Mey, K., Sterken, C. and Gillet, D.: 1995, Astron. Astrophys. 301, 781

Baade, D.: 1987, in A. Slettebak and Th.P. Snow (eds.): Physics of Be Stars, Proc. IAU Coll. No. 92, Cambridge Univ. Press, Cambridge, 361

Baade, D. and Balona, L.A.: 1994, in L.A. Balona, H.F. Henrichs, and J.M. Le Contel (eds.): Pulsation, Rotation, and Mass Loss in Early-type Stars, Proc. IAU Symp. No. 162, Kluwer, Dordrecht, 311

Balona, L.A.: 1992, Mon. Not. R. Astron. Soc. 256, 425

Balona, L.A.: 1993, Mon. Not. R. Astron. Soc. 260, 795

Balona, L.A., Dziembowski, W.A. and Pamyatnykh, A.A.: 1997, Mon. Not. R. Astron. Soc. 289, 25

Cassinelli, J.P., Cohen, D.H., MacFarlane, J.J., Drew, J.E., Lynas-Gray, A.E., Hubeny, I., Vallerga, J.V., Welsh, B.Y., Hoare, G.M.: Astrophys. J. 460, 949

Gies, D.R.: 1991, in D. Baade (ed.): Rapid Variability of OB-Stars: Nature and Diagnostic Value, ESO Workshop and Conf. Proc. No. 36, Europ. South. Observ. Garching, 229

Hadrava, P. and Harmanec, P.: 1996, Astron. Astrophys. 315, L401

Howarth, I.D. and Reid, A.H.N.: 1993, Astron. Astrophys. 279, 148

Jerzykiewicz, M. and Pigulski, A.: 1996, Mon. Not. R. Astron. Soc. 282, 853

Kambe, E., Ando, H., Hirata, R., Walker, G.A.H., Kennelly, E.J. and Matthews, J.M.: 1993, Publ. Astron. Soc. Pacific 105, 1222

Kambe, E., Hirata, R., Ando, H., Cuypers, J., Katoh, M., Kennelly, E.J., QWalker, G.A.H., Stefl, S. and Tarasov, A.E.: 1997, Astrophys. J. 481, 406

Kaufer, A., Stahl, O., Wolf, B., Gäng, Th., Gummersbach, C.A., Kovács, J., Mandel, H. and Szeifert, Th.: 1996, Astron. Astrophys. 305, 887

Kaufer, A., Stahl, O., Wolf, B., Fullerton, A.W., Gäng, Th., Gummersbach, C.A., Jankovics, I., Kovács, J., Mandel, H. Peitz, J., Rivinius, Th. and Szeifert, Th.: 1996, Astron. Astrophys. 320, 273

Reid, A.H.N. and Howarth, I.D.: 1996, Astron. Astrophys. 311, 616

Rivinius, Th., Baade, D., Štefl, S., Stahl, O., Wolf, B., Kaufer, A.: 1998, Astron. Astrophys., submitted

Smith, M.A.: 1981, in G.E.V.O.N. and C. Sterken (eds.): Workshop on Pulsating B Stars, Nice Obs., Nice, 317

Smith, M.A. and Penrod, D.G.: 1984, in R. Stalio and J. Zirker (eds.): Relat. between Chromosph.-coronal Heating and Mass Loss in Stars, Trieste, 394

Sterken, C.: 1993, in J.M. Nemec and J.M. Matthews (eds.): IAU Coll. No. 139, New Perspectives on Stellar Pulsation and Pulsating Variable Stars, Cambridge University Press, Cambridge, 171

Sterken, C. Jerzykiewicz, M.: 1993, $\beta$ Cephei Stars from a Photometric Point of View, Space Science Reviews 62, 95

Telting, J.H., Aerts, C. and Mathias, P.: 1997, Astron. Astrophys. 322, 493

van Leeuwen, F., van Genderen, A.M., Zegelaar, I.: 1998, submitted to Astron. Astrophys.

Waelkens, C.: 1991, Astron. Astrophys. 246, 453

Waelkens, C., Aerts, C., Kestens, E., Grenon, M., Eyer, L.: 1998, submitted to Astron. Astrophys. 